\title{
Occurrence and potentially zoonotic genotypes of Enterocytozoon bieneusi in wild rhesus macaques (Macaca mulatta) living in Nanwan Monkey Island, Hainan, China: a public health concern
}

Wei Zhao ${ }^{1,2}$, Huan-Huan Zhou ${ }^{1,3}$, Guang-Xu Ren ${ }^{1,4,5}$, Yu Qiang ${ }^{1,4,5}$, Hui-Cong Huang ${ }^{2}$, Gang Lu 1,4,5* $^{*}$ and Feng Tan ${ }^{2^{*}}$

\begin{abstract}
Background: Enterocytozoon bieneusi, a microsporidian species, is a zoonotic pathogen found in both humans and animals. Here, we determined the prevalence, explored the different genotypes of E. bieneusi in wild rhesus macaques (Macaca mulatta) (Hainan Island of China), and assessed their zoonotic potential.

Methods: We collected 173 fecal specimens from wild rhesus macaques living in Nanwan Monkey Island, Hainan, China. Subsequently, we identified and genotyped E. bieneusi using nested PCR analysis amplification of the internal transcribed spacer region (ITS) of the rRNA gene. Lastly, a neighbor-joining tree was built based on gene sequences from the ITS region of E. bieneusi.

Results: Of the 173 specimens from wild rhesus macaques, 26 (15\%) were infected with E. bieneusi. We identified six genotypes of E. bieneusi, of which five were known: PigEBITS7 $(n=20)$, D $(n=2)$, Type IV $(n=1)$, Peru6 $(n=1)$, Henan-III $(n=1)$, and a novel genotype: HNM-IX $(n=1)$. From the phylogenetic analysis, the six genotypes identified here were all clustered into zoonotic group 1.
\end{abstract}

Conclusion: This study is the first report to detect E. bieneusi infection in wild rhesus macaques from Hainan, China. Human-pathogenic genotypes D, Henan-III, Peru6, PigEbITS7, and Type IV in the wild rhesus macaques support these animals infected with $E$. bieneusi have a public health significance.

Keywords: Enterocytozoon bieneusi, Rhesus macaques, Hainan, Zoonotic

\footnotetext{
*Correspondence: luganghn@163.com; tanfengsong@163.com

'Key Laboratory of Tropical Translational Medicine of Ministry of Education, Hainan Medical University, Haikou 571199, China

${ }^{2}$ Department of Parasitology, Wenzhou Medical University, Wenzhou 325035,

Zhejiang, China

Full list of author information is available at the end of the article
}

(c) The Author(s). 2021 Open Access This article is licensed under a Creative Commons Attribution 4.0 International License, which permits use, sharing, adaptation, distribution and reproduction in any medium or format, as long as you give appropriate credit to the original author(s) and the source, provide a link to the Creative Commons licence, and indicate if changes were made. The images or other third party material in this article are included in the article's Creative Commons licence, unless indicated otherwise in a credit line to the material. If material is not included in the article's Creative Commons licence and your intended use is not permitted by statutory regulation or exceeds the permitted use, you will need to obtain permission directly from the copyright holder. To view a copy of this licence, visit http://creativecommons.org/licenses/by/4.0/ The Creative Commons Public Domain Dedication waiver (http://creativecommons.org/publicdomain/zero/1.0/) applies to the data made available in this article, unless otherwise stated in a credit line to the data. 


\section{Background}

Enterocytozoon bieneusi is a typical human-pathogenic microsporidia species that invades the enterocytes of the small intestine [1]. Although its general infection is described by chronic diarrhea, and malabsorption or no clinical signs in immunocompetent humans, it can result in enhanced increased fatality via chronic diarrhea in individuals with immunodeficiency, such as patients with Acquired Immune Deficiency Syndrome (AIDS) [2]. Different studies have shown that it appears in several animals (mammals, birds, and reptiles) and some environmental samples (water, soil, and food) [3]. Most human infections result from the zoonotic transmission of spores through either contaminated food or water [4].

Recent surveys incorporated genotype information of E. bieneusi and elaborated genotype distribution among human populations and animal hosts [3]. Different studies have observed substantial genetic diversity within this species through sequencing the single internal transcribed spacer (ITS) region of the rRNA gene [5]. To date, scientists have detected approximately $600 \mathrm{E}$. bieneusi ITS genotypes [6]. Among these genotypes, 49 were found in both animals and humans [3]. All genotypes of E. bieneusi could be categorized into 13 clades [7]. Here, two large groups (1 and 2 ) that are composed of genotypes common in animals and humans are termed zoonotic. The remaining 11 groups (3 to 13) contain genotypes from specific hosts or wastewater [3]. Furthermore, $E$. bieneusi is commonly detected in various wildlife and a wide variety of both potentially host-adapted and zoonotic genotypes have been identified [3, 8]. Thus, wildlife is also an ecological resource for several human/ animal infections. Therefore, the primary focus of epidemiological surveys should involve the genotyping of $E$. bieneusi isolates from under-sampled animal hosts with human contact to expand our knowledge regarding human microsporidiosis epidemiology and support E. bieneusi population analysis.

Rhesus macaques (Macaca mulatta) are prevalent in Southeast Asia, where their geographic range overlaps extensively with that of humans [9]. We carried out this study in the Nanwan Monkey Island, Nanwan peninsula, Lingshui county, south coast of Hainan, China. Globally, this is the only island-type nature reserve for rhesus macaques and is home to over 2500 monkeys. This island has a primitive natural environment, which makes it a perfect place for monkeys. Since its establishment in
1965, it has become a popular tourist destination. However, there is a lack of published studies on E. bieneusi infection in the rhesus macaques living in the Nanwan Monkey Island. Therefore, the aim of this study was to investigate the incidence and different genotypes of $E$. bieneusi present in the wild rhesus macaques.

\section{Results}

\section{Infection rates of $E$. bieneusi in wild $M$. mulatta}

Twenty-six of 173 specimens from wild rhesus macaques were positive for E. bieneusi since they amplified the ITS region of the rRNA gene, with an average infection rate of $15.0 \%$. Besides, the infection rate of E. bieneusi in rhesus macaques less than 1 year of age $(19.4 \%$; 14/72) was higher than those animals older than 1 year (11.9\%; 12 / 101) (Table 1). Meanwhile, out of all the positives, $14.2 \%$ $(17 / 120)$ were females, and $17.0 \%(9 / 53)$ were males (Table 2). However, as illustrated in Tables 1 and 2, the infection rates difference were not statistically significant either by age or by gender.

\section{Genotype distribution of $E$. bieneusi by gender and age} Six genotypes were identified in the wild rhesus macaques through sequencing and multiple sequence alignment. They included five known genotypes (D, HenanIII, Peru6, PigEbITS7, and Type IV) and one novel genotype (HNM-IX). Among them, genotype PigEbITS7 was dominant, and was found in $76.9 \%(20 / 26)$ of E. bieneusi isolates. All the remaining genotypes were at a lower frequency: $7.7 \%(2 / 26)$ for genotype $\mathrm{D}$, and $3.8 \%(1 / 26)$ each for genotypes Peru 6, Type IV, Henan-III, and HNM-IX. Subsequently, two genotypes (D and PigEbITS7) were detected in the less than one-year-old animals, whereas five genotypes (Henan-III, HNM-IX, Peru 6, PigEbITS7 and Type IV) in the more than one-yearold animals (Table 1). As demonstrated in Table 2, two genotypes (PigEbITS7 and Type IV) were predominant in males, whereas five (D, Henan-III, HNM-IX, PigEbITS7, and Peru 6) in females.

\section{Genetic relationships of ITS genotypes \\ Novel genotype HNM-IX had one single nucleotide polymorphism (SNP), with genotype EbpC (AF076042) having it at nucleotide site 51 of the ITS region. As illus- trated in Fig. 1, phylogenetic analysis revealed that all genotypes belonged to zoonotic group 1 . They were fur- ther sub-divided into different genotype sub-groups such}

Table 1 Prevalences of E. bieneusi and distributions of genotypes in Macaca mulatta by age

\begin{tabular}{llll}
\hline Age (Years) & Positive no./Examined no.(\%) & Genotype(s) (n) & Statistics value \\
\hline Less than one & $14 / 72(19.4)$ & PigEbITS7(12); D(2) & $X^{2}=1.88, P=0.17$ \\
Over one & $12 / 101(11.9)$ & PiglTS7(8); Peru 6 (1); Type IV (1); HNM-IX (1); Henan-III (1) & \\
Total & 26/173 (15.0) & PigITS7(12); D(2); Peru 6 (1); Henan-III (1); HNM-IX (1); Type IV (1) & \\
\hline
\end{tabular}


Table 2 Prevalences of E. bieneusi and distributions of genotypes in Macaca mulatta by gender

\begin{tabular}{llll}
\hline Gender & Positive no./Examined no.(\%) & Genotype(s) (n) & Statistics value \\
\hline Male & $9 / 53(17.0)$ & PiglTS7 (8); Type IV (1) & $X^{2}=0.23, P=0.63$ \\
Female & $17 / 120(14.2)$ & PiglTS7(12); D(2); Peru 6 (1); HNM-IX (1); Henan-III (1) & \\
Total & 26/173 (15.0) & PigITS7(12); D(2); Peru 6 (1); Henan-III (1); HNM-IX (1); Type IV (1) & \\
\hline
\end{tabular}

as PigEbITS7 and D in subgroup1a; genotypes Peru 6 in subgroup 1b; genotype Type IV in subgroup 1c; and genotypes Henan-III and HNM-IX in subgroup 1d.

\section{Discussion}

Non-human primates (NHPs) are known to possess a high genetic relationship with humans, which makes them useful biomedical research models. NHPs might be vulnerable to human diseases, thereby acting as zoonotic reservoirs, such as Cryptosporidium, Giardia, Blastocystis, etc. $[9,11]$. In 1997, the first case of transference of E. bieneusi infection was recorded between a human (afflicted with AIDS) and a rhesus monkey (afflicted with simian immunodeficiency virus) [12]. However, until 2011 , there was a lack of studies on the occurrence of $E$. bieneusi in non-human primates at the genotype level [11]. Zhao et al., summarized 16 studies on the infection of E. bieneusi in NHPs from seven countries [9, 13].
Among them, seven studies included rhesus macaques, and they were all from China, with a prevalence range from 4.2 to $31.1 \%$ [13-18]. For the first time, our study has detected $E$. bieneusi in wild rhesus macaques from the Hainan Province of China, with a prevalence of $15.0 \%$. Generally, E. bieneusi has been found to be more prevalent in wild rhesus macaques here than other wild NHPs, such as baboons from Kenya (12.3\%) [11], chimpanzees from Cameroon (4.5\%) and Kenya (2.6\%) [19], gorillas from the Central African Republic (4.0\%) [20], orangutans from Indonesia (2.0\%) [21], and five captive species of wild NHPs from the Qinling Mountains of China [17]. Our study showed that E. bieneusi was more prevalent in rhesus macaques than farm monkeys from Henan (6.8\%), Guangxi (8.5\%), Sichuan (10.5\%), and zoo monkeys from Henan (12.5\%) in China [15, 16, 22]. However, the prevalence of E. bieneusi in monkeys from Rwanda (18.0\%) and some cities in China, like Shanxi

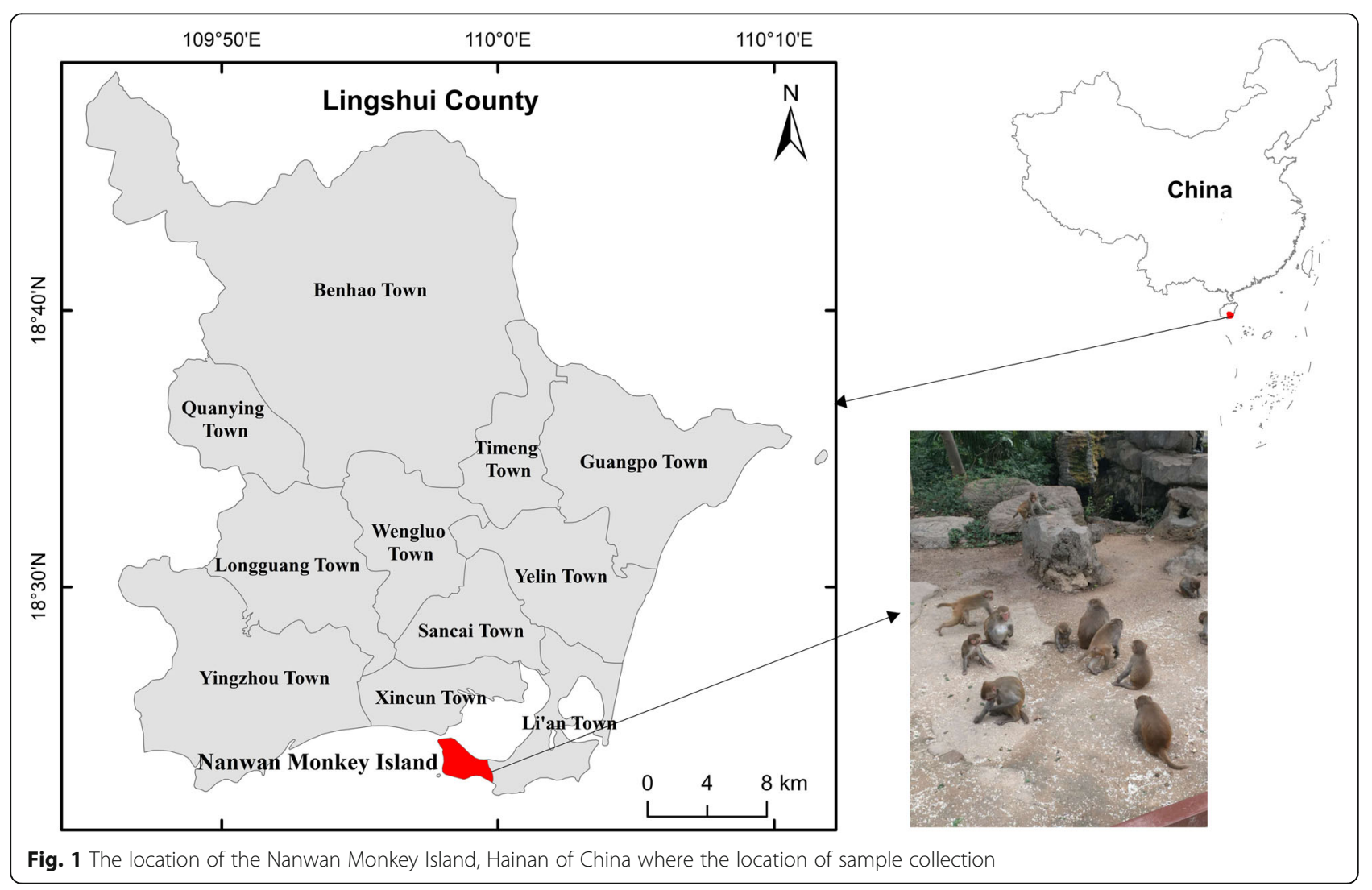


(18.2\%), Shanghai (26.7\%), Hebei (27.0\%), and Beijing (29.2\%) was higher than that observed in our study [15, $16,20,22]$. Additionally, there are two more studies that identified $E$. bieneusi infection in laboratory macaques in Beijing (25.6\%) and Guangxi (18.5\%), China, which were both higher compared with this study [14, 23]. In fact, in Hainan, two studies were reported on captive long-tailed macaques infected with $E$. bieneusi, which were also more than that observed in our study $[9,24]$. Similar to humans and farm animals, age substantially increases the risk of E. bieneusi infection in NHPs [9]. Here, we identified a elevated $E$. bieneusi infection rate in young rhesus macaques compared with adults, which agreed with the results of captive long-tailed macaque and laboratory macaques from Hainan, China and North China, respectively $[9,15]$. In addition to age, the health of the hosts, the detection methods, sample size, the experimental design, animal practices, etc. could cause the increase in prevalence.

Among the five known genotypes in our study, the genotype PigEbITS7 was detected in 76.9\% (20/26) of $E$. bieneusi isolates, which shows predominance in the investigated wild rhesus macaques. This genotype was initially detected in pigs from the USA [8] and it has been confirmed to have a broad host range, even in humans [3]. In China, PigEBITS7 was detected in some patients, including AIDS and hospitalized children, and several animals such as rodents, NHPs, and urban wastewater [5, 9, 25-27]. Notably, genotype PigEBITS7 is also a common genotype in wild rodents such as asian house rats, brown rats and Chinese white-bellied rats, so this genotype may transmission from infected rodents to macaques [9].

Additionally, previous studies have reported the presence of four other genotypes (D, Henan-III, Peru6, and Type IV) in humans and animals around the world, of which genotypes D and Type IV are commonly found in E. bieneusi-induced microsporidiosis in humans [3, 28]. Both genotypes D and Type IV have been detected in infants, HIV-positive patients, and HIV-negative patients in China [25, 29-33]. Meanwhile, they have been found in NHPs, pigs, dogs, snakes, cats, hippopotamus, Pere David's deer, chinchillas, Siberian tiger, lions, Fischer's lovebird, red foxes, wastewater, and lake water [3]. In addition, genotype $\mathrm{D}$ has also recorded in other animals, such as bear, bornean orangutan, cattle, common crane, dog, goat, golden takin, golden snub-nosed monkey, hamadryas baboon, horse, rabbit, rat, raccoon, sheep [6]. Genotypes Peru 6 (syn. PtEbI, PtEbVII) (from Peru and Portugal) and Henan-III (from Malaysia and China) have been spread across limited geographical area as well as small number of E. bieneusi-infected human cases compared with genotypes D and Type I [34-36]. Meanwhile, genotype Peru 6 has been identified in sheep, goats, reindeers, and wastewater [37-40], whereas genotype Henan-III has been found in NHPs, pet snakes, pigs, and birds in China [41-44]. Therefore, the above shreds of evidence suggest the possible zoonotic transmission of these genotypes from the wild rhesus macaques to humans.

In this study, the novel genotype HNM-IX was genetically closely related to the human-pathogenic genotype $\mathrm{EbpC}$ which was commonly found in humans from Iran, Czech Republic, Peru, China, Thailand, and Vietnam [29, 45-48]. It was also found in more than 15 animal species and in environmental samples $[3,10]$. From the phylogenetic analysis, the six genotypes identified here were all categorized into group 1. Group 1 had almost all human-pathogenic genotypes and possessed $94 \%$ of the known E. bieneusi ITS sequences [3]. Therefore, the genotypes in wild macaques investigated including the novel one could have a sizeable zoonotic possibility.

\section{Conclusions}

This study is the first report to detect $E$. bieneusi infection in wild rhesus macaques from Hainan, China. Humanpathogenic genotypes D, Henan-III, Peru6, PigEbITS7, and Type IV in these animals support a zoonotic nature for E. bieneusi. Here, phylogenetic analysis showed that the novel genotype fell into group 1, suggesting its zoonotic possibility. Thus, visitors, veterinary workers, and the management of the wild rhesus macaques should be educated and informed to minimize the risk for transmission of $E$. bieneusi from those animals.

\section{Methods}

\section{Fecal sample collection}

We obtained 173 stool samples from rhesus macaques in Nanwan Monkey Island, located on the Nanwan peninsula, Lingshui county, south coast of Hainan, in the southernmost province of China. It is geographically located at $109^{\circ} 48^{\prime}$ east longitude and $18^{\circ} 29^{\prime}$ north latitude (Fig. 2). In this study, the sampled macaques were from six major scenic areas of this island and approximately $30 \%$ of the total macaques of each areas were collected. Under the help of the trainer, we used sterile disposable latex gloves to collect fresh fecal samples, which were then placed in plastic cups marked with the collect date and the macaque's age and sex. All specimens were transported to our laboratory and stored at $4{ }^{\circ} \mathrm{C}$.

\section{DNA extraction}

We sieved the fecal specimens, concentrated the filtrates, washed them thrice with distilled water, and centrifuged (10 min, 1500 g). Then, we used a QIAamp DNA Stool Mini Kit (Qiagen, Hilden, Germany) to extract genomic DNA from washed fecal specimens (180-200 mg) 


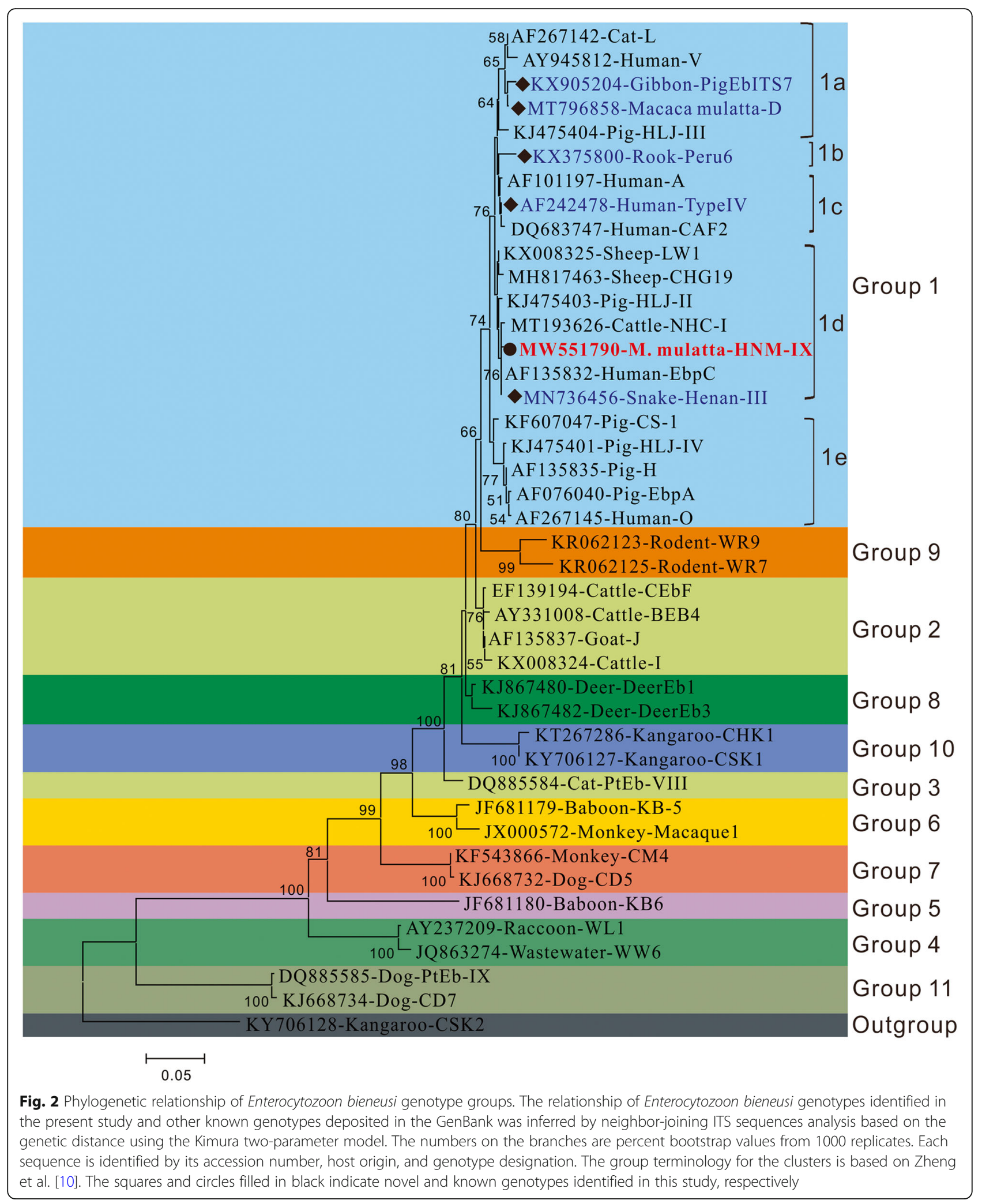


following manufacturer's guidelines. Finally, DNA was eluted in $200 \mu \mathrm{L}$ of $\mathrm{AE}$ buffer and stored at $-20^{\circ} \mathrm{C}$.

\section{PCR amplification}

We analyzed all DNA preparations for E. bieneusi using nested PCR amplification. This amplification contained a nucleotide fragment (389 bp) containing 3' end small subunit $(\mathrm{SSU})(76 \mathrm{bp})$, ITS region $(243 \mathrm{bp})$, and $5^{\prime}$ region of the large subunit (LSU) $(70 \mathrm{bp})$ from E. bieneusi rRNA gene. Primers and cycle parameters were designed by Buckholt et al. [49]. Specifically, two pairs of primers, including EBITS3 and EBITS4 and EBITS1 and EBITS2.4, were used in the first and the second PCR amplification processes, producing nucleotide fragments of 435 and $389 \mathrm{bp}$, respectively. The two cycling parameters used for PCR reactions were as follows: 35 cycles of $94^{\circ} \mathrm{C}$ for $30 \mathrm{~s}, 57^{\circ} \mathrm{C}$ for $30 \mathrm{~s}$, and $72{ }^{\circ} \mathrm{C}$ for $40 \mathrm{~s}$, and 30 cycles of $94^{\circ} \mathrm{C}$ for $30 \mathrm{~s}$, $55^{\circ} \mathrm{C}$ for $30 \mathrm{~s}$, and $72^{\circ} \mathrm{C}$ for $40 \mathrm{~s}$, with both of them having a final extension step at $72{ }^{\circ} \mathrm{C}$ for $10 \mathrm{~min}$. The TaKaRa Taq DNA Polymerase (TaKaRa Bio Inc., Tokyo, Japan) was used for all PCR amplifications. Meanwhile, all PCR amplification tests were carried out with positive controls (cattle-derived genotype BEB4 DNA) as well as a negative control (no DNA). Finally, all PCR products were separated via $1.5 \%$ agarose gel electrophoresis, followed by ethidium bromide staining.

\section{Nucleotide sequencing}

All appropriately sized PCR products were purified using the Big Dye Terminator v3.1 Cycle Sequencing Kit (Applied Biosystems, USA) on an ABI PRISM 3730 XL DNA Analyzer (Sinogeno- max Biotechnology Co. Ltd., Beijing, China), followed by direct sequencing using PCR primers. We performed bidirectional sequencing to verify sequence accuracy.

\section{Sequence analysis}

We determined E. bieneusi genotypes. Here, we aligned the nucleotide sequences with each other, and used the BLAST and Clustal X 1.83 to access the reference sequences. In particular, the first published names corresponded to the sequences who had a 100\% resemblance to those from known genotypes. Otherwise, they were described as novel genotypes. Finally, the nomenclature was established by naming all genotypes according to the $243 \mathrm{bp}$ of the ITS gene region of E. bieneusi [5].

\section{Phylogenetic analysis}

Here, we studied the genetic association between the novel and known genotypes. Next, we used the Mega X software (http://www.megasoftware.net/) to compare the ITS region of all identified nucleotide sequences with those of the reference sequences. The neighbor-joining tree was built based on the evolutionary distances calculated using a Kimura 2parameter model and bootstrap analysis of 1000 replicates.

\section{Abbreviations}

AIDS: acquired immune deficiency syndrome; SNP: single nucleotide polymorphism; NHPs: non-human primates; BLAST: Basic Local Alignment Search Tool; ITS: internal transcribed spacer; SSU: small subunit; LSU: large subunit

\section{Acknowledgements}

We thank Editideas (www.editideas.cn) for its linguistic assistance during the preparation of this manuscript.

\section{Authors' contributions}

WZ, FT and GL conceived the study and contributed to the design. $\mathrm{H}-\mathrm{H} Z$ and WZ contributed to acquisition of samples. $\mathrm{H}-\mathrm{H} Z$ Z, G-X R and YQ performed experiments. WZ, $\mathrm{H}-\mathrm{H}$ Z and $\mathrm{H}-\mathrm{C} \mathrm{H}$ contributed to data analysis. WZ contributed to writing the manuscript. FT and GL review and editing the manuscript. All authors approved the final version to be published and agreed to be accountable for all aspects of the manuscript.

\section{Funding}

This work was supported by the Major Science and Technology Program of Hainan Province (ZDKJ202003); Research project of Hainan academician innovation platform (YSPTZX202004); Hainan talent development project (SRC200003); the National Natural Science Foundation of China

(No.82060375); Innovation Research Team Project of Hainan Natural Science Foundation (2018CXTD340) and the Open Foundation of Key Laboratory of Tropical Translational Medicine of Ministry of Education, Hainan Medical University (2020TTM004).

\section{Availability of data and materials}

All data generated or analysed during this study are included in this published article. The identified nucleotide sequence of the novel genotype was submitted to the GenBank database (accession\# MW551790).

\section{Declarations}

Ethics approval and consent to participate

The Research Ethics Committee and the Animal Ethics Committee of Hainan Medical University approved the study protocol. All animal experiments complied with the guidelines provided by the Regulations for the Administration of Affairs Concerning Experimental Animals.

\section{Consent for publication}

Not applicable.

\section{Competing interests}

The authors declare that they have no competing interests.

\section{Author details}

${ }^{1}$ Key Laboratory of Tropical Translational Medicine of Ministry of Education, Hainan Medical University, Haikou 571199, China. ${ }^{2}$ Department of Parasitology, Wenzhou Medical University, Wenzhou 325035, Zhejiang, China. ${ }^{3}$ Qingdao Shinan District Center for Disease Control and Prevention, Qingdao 266071, Shandong, China. ${ }^{4}$ Department of Pathogenic Biology, Hainan Medical University, Haikou, Hainan, China. ${ }^{5}$ Hainan Medical University-The University of Hong Kong Joint Laboratory of Tropical Infectious Diseases, Hainan Medical University, Haikou, Hainan, China.

Received: 12 February 2021 Accepted: 21 May 2021

Published online: 09 June 2021

\section{References}

1. Fadhilah A, Gabbar A, Bokhari AA. Microsporidium. In: StatPearls. Treasure Island: StatPearls Publishing; 2020.

2. Li W, Xiao L. Ecological and public health significance of Enterocytozoon bieneusi. One Health. 2020;12:100209.

3. Li W, Feng Y, Santin M. Host specificity of Enterocytozoon bieneusi and public health implications. Trends Parasitol. 2019;35:436-51.

4. Fayer R, Santin-Duran M. Epidemiology of microsporidia in human infections. In: Weiss LM, Becnel JJ, editors. Microsporidia: Pathogens of Opportunity. 1st ed. Chichester: Wiley; 2024. p. 1-64. https://doi.org/10.1 002/9781118395264. 
5. Santín M, Fayer R. Enterocytozoon bieneusi genotype nomenclature based on the internal transcribed spacer sequence: a consensus. J Eukaryot Microbiol. 2009;56:34-8.

6. Zhang Y, Koehler AV, Wang T, Gasser RB. Enterocytozoon bieneusi of animalswith an 'Australian twist'. Adv Parasitol 2021;111:1-73.

7. Zhao W, Zhou H, Yang L, Ma T, Zhou J, Liu H, et al. Prevalence, genetic diversity and implications for public health of Enterocytozoon bieneusi in various rodents from Hainan Province, China. Parasit Vectors. 2020;13:438.

8. Leśniańska K, Perec-Matysiak A. Wildlife as an environmental reservoir of Enterocytozoon bieneusi (Microsporidia) - analyses of data based on molecular methods. Ann Parasitol. 2017;63:265-81.

9. Zhao W, Zhou H, Jin H, Sun L, Li P, Liu M, et al. Genotyping of Enterocytozoon bieneusi among captive long-tailed macaques (Macaca fascicularis) in Hainan Province: high genetic diversity and zoonotic potential. Acta Trop. 2020;201:105211.

10. Zheng XL, Zhou HH, Ren G, Ma TM, Cao ZX, Wei LM, et al. Genotyping and zoonotic potential of Enterocytozoon bieneusi in cattle farmed in Hainan Province, the southernmost region of China. Parasite. 2020;27:65.

11. Li W, Kiulia NM, Mwenda JM, Nyachieo A, Taylor MB, Zhang X, et al. Cyclospora papionis, Cryptosporidium hominis, and human-pathogenic Enterocytozoon bieneusi in captive baboons in Kenya. J Clin Microbiol. 2011; 49:4326-9.

12. Tzipori S, Carville A, Widmer G, Kotler D, Mansfield K, Lackner A. Transmission and establishment of a persistent infection of Enterocytozoon bieneusi, derived from a human with AIDS, in simian immunodeficiency virus-infected rhesus monkeys. J Infect Dis. 1997;175:1016-20.

13. Yu M, Liu X, Xie M, Li D, Ni Q, Zhang M, et al. Epidemiological investigation and genotypes of Enterocytozoon bieneusi in 11 captive rhesus macaque populations. Int J Parasitol Parasites Wildl. 2020;13:191-5.

14. Yang H, Lin Y, Li Y, Song M, Lu Y, Li W. Molecular characterization of Enterocytozoon bieneusi isolates in laboratory macaques in North China: zoonotic concerns. Parasitol Res. 2017:116:2877-82.

15. Zhong Z, Li W, Deng L, Song Y, Wu K, Tian Y, et al. Multilocus genotyping of Enterocytozoon bieneusi derived from nonhuman primates in Southwest China. PLoS One. 2017;12:e0176926.

16. Karim MR, Wang R, Dong H, Zhang L, Li J, Zhang S, et al. Genetic polymorphism and zoonotic potential of Enterocytozoon bieneusi from nonhuman primates in China. Appl Environ Microbiol. 2014;80:1893-8.

17. Du SZ, Zhao GH, Shao JF, Fang YQ, Tian GR, Zhang LX, et al. Cryptosporidium spp., Giardia intestinalis, and Enterocytozoon bieneusi in captive non-human primates in Qinling Mountains. Korean J Parasitol. 2015; 53:395-402

18. Ye J, Xiao L, Ma J, Guo M, Liu L, Feng Y. Anthroponotic enteric parasites in monkeys in public park, China. Emerg Infect Dis. 2012;18:1640-3.

19. Sak B, Kvác M, Petrzelková K, Kvetonová D, Pomajbíková K, Mulama M, et al. Diversity of microsporidia (Fungi: Microsporidia) among captive great apes in European zoos and African sanctuaries: evidence for zoonotic transmission? Folia Parasitol (Praha). 2011;58:81-6.

20. Sak B, Petrzelkova KJ, Kvetonova D, Mynarova A, Shutt KA, Pomajbikova K, et al. Long-term monitoring of microsporidia, Cryptosporidium and Giardia infections in western lowland gorillas (Gorilla gorilla gorilla) at different stages of habituation in Dzanga Sangha protected areas, Central African Republic. PLoS One. 2013;8:e71840.

21. Mynářová A, Foitová I, Kváč $M$, Květoňová $D$, Rost $M$, Morrogh-Bernard $H$, et al. Prevalence of Cryptosporidium spp., Enterocytozoon bieneusi, Encephalitozoon spp. and Giardia intestinalis in wild, semi-wild and captive orangutans (Pongo abelii and Pongo pygmaeus) on Sumatra and Borneo, Indonesia. PLoS One. 2016;11:e0152771.

22. Yu F, Wu Y, Li T, Cao J, Wang J, Hu S, et al. High prevalence of Enterocytozoon bieneusi zoonotic genotype D in captive golden snub-nosed monkey (Rhinopithecus roxellanae) in zoos in China. BMC Vet Res. 2017;13:158.

23. Ye J, Xiao L, Li J, Huang W, Amer SE, Guo Y, et al. Occurrence of humanpathogenic Enterocytozoon bieneusi, Giardia duodenalis and Cryptosporidium genotypes in laboratory macaques in Guangxi, China. Parasitol Int. 2014;63: 132-7.

24. Chen L, Zhao J, Li N, Guo Y, Feng Y, Feng Y, et al. Genotypes and public health potential of Enterocytozoon bieneusi and Giardia duodenalis in crabeating macaques. Parasit Vectors. 2019;12:254.

25. Yu F, Li D, Chang Y, Wu Y, Guo Z, Jia L, et al. Molecular characterization of three intestinal protozoans in hospitalized children with different disease backgrounds in Zhengzhou, Central China. Parasit Vectors. 2019;12:543.
26. Liu H, Jiang Z, Yuan Z, Yin J, Wang Z, Yu B, et al. Infection by and genotype characteristics of Enterocytozoon bieneusi in HIV/AIDS patients from Guangxi Zhuang autonomous region, China. BMC Infect Dis. 2017;17:684.

27. Li N, Xiao L, Wang L, Zhao S, Zhao X, Duan L, et al. Molecular surveillance of Cryptosporidium spp., Giardia duodenalis, and Enterocytozoon bieneusi by genotyping and subtyping parasites in wastewater. PLoS Negl Trop Dis. 2012;6:e1809

28. Matos O, Lobo ML, Xiao L. Epidemiology of Enterocytozoon bieneusi infection in humans. J Parasitol Res. 2012;2012:981424.

29. Wang L, Zhang H, Zhao X, Zhang L, Zhang G, Guo M, et al. Zoonotic Cryptosporidium species and Enterocytozoon bieneusi genotypes in HIVpositive patients on antiretroviral therapy. J Clin Microbiol. 2013;51:557-63.

30. Zang M, Li J, Tang C, Ding S, Huang W, Qin Q, et al. Prevalence and phylogenetic analysis of Microsporidium Enterocytozoon bieneusi in diarrheal patients. Pathogens. 2021;10:128.

31. Qi M, Yu F, Zhao A, Zhang Y, Wei Z, Li D, et al. Unusual dominant genotype NIA1 of Enterocytozoon bieneusi in children in southern Xinjiang, China. PLoS Negl Trop Dis. 2020;14:e0008293.

32. Zhang W, Ren G, Zhao W, Yang Z, Shen Y, Sun Y, et al. Genotyping of Enterocytozoon bieneusi and subtyping of Blastocystis in cancer patients: relationship to diarrhea and assessment of zoonotic transmission. Front Microbiol. 2017:8:1835

33. Wang T, Fan Y, Koehler AV, Ma G, Li T, Hu M, et al. First survey of Cryptosporidium, Giardia and Enterocytozoon in diarrhoeic children from Wuhan, China. Infect Genet Evol. 2017;51:127-31.

34. Sulaiman IM, Bern C, Gilman R, Cama V, Kawai V, Vargas D, et al. A molecular biologic study of Enterocytozoon bieneusi in HIV-infected patients in Lima, Peru. J Eukaryot Microbiol. 2003;50(Suppl):591-6.

35. Gong B, Yang Y, Liu X, Cao J, Xu M, Xu N, et al. First survey of Enterocytozoon bieneusi and dominant genotype Peru6 among ethnic minority groups in southwestern China's Yunnan Province and assessment of risk factors. PLoS Negl Trop Dis. 2019;13:e0007356.

36. Ruviniyia K, Abdullah DA, Sumita S, Lim YAL, Ooi PT, Sharma RSK. Molecular detection of porcine Enterocytozoon bieneusi infection in peninsular Malaysia and epidemiological risk factors associated with potentially zoonotic genotypes. Parasitol Res. 2020;119:1663-74.

37. Zhao W, Yu S, Yang Z, Zhang Y, Zhang L, Wang R, et al. Genotyping of Enterocytozoon bieneusi (Microsporidia) isolated from various birds in China. Infect Genet Evol. 2016;40:151-4.

38. Zhao W, Zhang W, Yang D, Zhang L, Wang R, Liu A. Prevalence of Enterocytozoon bieneusi and genetic diversity of ITS genotypes in sheep and goats in China. Infect Genet Evol. 2015;32:265-70.

39. Liu W, Nie C, Zhang L, Wang R, Liu A, Zhao W, et al. First detection and genotyping of Enterocytozoon bieneusi in reindeers (Rangifer tarandus): a zoonotic potential of ITS genotypes. Parasit Vectors. 2015;8:526.

40. Ye J, Ji Y, Xu J, Ma K, Yang X. Zoonotic Enterocytozoon bieneusi in raw wastewater in Zhengzhou, China. Folia Parasitol (Praha). 2017;64:2017. 002.

41. Dong H, Cheng R, Li X, Li J, Chen Y, Ban C, et al. Molecular Identification of Cryptosporidium spp., Enterocytozoon bieneusi, and Giardia duodenalis in captive pet birds in Henan province, central China. J Eukaryot Microbiol. 2021:e12839.

42. Li D, Zheng S, Zhou C, Karim MR, Wang L, et al. Multilocus typing of Enterocytozoon bieneusi in pig reveals the high prevalence, zoonotic potential, host adaptation and geographical segregation in China. J Eukaryot Microbiol. 2019;66:707-18.

43. Li J, Li D, Zhang H, Wang R, Lin Z, Zhang L, et al. Molecular characterization and novel genotypes of Enterocytozoon bieneusiin pet snakes in Beijing, China. Int J Parasitol Parasites Wildl. 2020;12:172-5.

44. Wang Y, Zhang K, Zhang Y, Wang K, Gazizova A, Wang L, et al. First detection of Enterocytozoon bieneusi in whooper swans (Cygnus cygnus) in China. Parasit Vectors. 2020;13:5.

45. Espern A, Morio F, Miegeville M, Illa H, Abdoulaye M, Meyssonnier V, et al. Molecular study of microsporidiosis due to Enterocytozoon bieneusi and Encephalitozoon intestinalis among human immunodeficiency virus-infected patients from two geographical areas: Niamey, Niger, and Hanoi, Vietnam. J Clin Microbiol. 2007:45:2999-3002.

46. Leelayoova S, Subrungruang I, Suputtamongkol Y, Worapong J, Petmitr PC, Mungthin M. Identification of genotypes of Enterocytozoon bieneusi from stool samples from human immunodeficiency virus-infected patients in Thailand. J Clin Microbiol. 2006;44:3001-4. 
47. Mirjalali H, Mirhendi H, Meamar AR, Mohebali M, Askari Z, Mirsamadi ES, et al. Genotyping and molecular analysis of Enterocytozoon bieneusi isolated from immunocompromised patients in Iran. Infect Genet Evol. 2015;36:244-9.

48. Yang J, Song M, Wan Q, Li Y, Lu Y, Jiang Y, et al. Enterocytozoon bieneusi genotypes in children in Northeast China and assessment of risk of zoonotic transmission. J Clin Microbiol. 2014;52:4363-7.

49. Buckholt MA, Lee JH, Tzipori S. Prevalence of Enterocytozoon bieneusi in swine: an 18-month survey at a slaughterhouse in Massachusetts. Appl Environ Microbiol. 2002;68:2595-9.

\section{Publisher's Note}

Springer Nature remains neutral with regard to jurisdictional claims in published maps and institutional affiliations.

Ready to submit your research? Choose BMC and benefit from:

- fast, convenient online submission

- thorough peer review by experienced researchers in your field

- rapid publication on acceptance

- support for research data, including large and complex data types

- gold Open Access which fosters wider collaboration and increased citations

- maximum visibility for your research: over $100 \mathrm{M}$ website views per year

At BMC, research is always in progress.

Learn more biomedcentral.com/submissions 